\title{
L'IDEA DI EQUITÀ NELLE TEORIE DELLA GIUSTIZIA
}

\author{
SALVATORE VECA $(*)$
}

SuNTO. - L'autore esamina il ruolo che l'idea di equità svolge entro le teorie della giustizia sociale. Riferendosi al fondamentale contributo teorico di John Rawls, mette a fuoco le relazioni fra i due principi della giustizia come equità e propone un'interpretazione che connette un argomento intuitivo a favore dei principi con l'argomento analitico e la teoria della scelta collettiva. Infine, accenna ai casi di estensione della giustizia come equità sottolineando, in particolare, l'importanza e le difficoltà di un'idea coerente di giustizia globale.

$$
* * *
$$

ABSTRACT. - The author examines the role that the idea of fairness plays in theories of social justice. Referring to the fundamental theoretical contribution of John Rawls, he focuses on the connections between the two principles of justice as fairness and offers an interpretation that combines an intuitive argument in favor of the principles with an analytical argument and the theory of collective choice. In conclusion, he cites cases of the extension of the concept of justice as fairness, stressing in particular the importance of and difficulty in establishing a coherent idea of global justice.

Nel mio intervento mi propongo di esaminare e illustrare l'idea di equità entro il quadro delle teorie della giustizia, intese come teorie politiche normative. Mi riferisco, in particolare, alla proposta teorica di John Rawls, formulata nel suo classico A Theory of Justice (1971), un

(*) Istituto Lombardo Accademia di Scienze e Lettere; IUSS Pavia, Italia. E-mail: Salvatore.veca@iusspavia.it 
contributo che ha istituito il paradigma delle teorie della giustizia entro la filosofia politica contemporanea ${ }^{1}$.

Com'è noto, l'idea principale di Rawls è che il ricorso alla nozione di equità (fairness) consenta di specificare i tratti fondamentali di una interpretazione della giustizia sociale. Il concetto di giustizia può infatti essere interpretato in modi differenti. Nel gergo di Rawls, un concetto di giustizia, più concezioni della stessa. L'interpretazione favorita da $A$ Theory of Justice è definita dalla concezione della giustizia come equità sociale.

I principi della giustizia come equità sono due. Il primo prescrive che ciascun partner della polis disponga di un eguale sistema delle libertà fondamentali (principio di libertà). Il secondo regola la distribuzione di costi e benefici della cooperazione sociale e prescrive che le uniche ineguaglianze giustificabili, quanto all'accesso a beni sociali primari, siano quelle che vanno a vantaggio dei gruppi meno avvantaggiati nella società in presenza di equa eguaglianza delle opportunità (principio di differenza).

Giustizia come equità è presentata da Rawls nei termini di una teoria del contratto sociale. I due principi di giustizia sono l'esito di una scelta collettiva (contratto sociale) in una opportuna situazione iniziale di scelta, interpretata come una posizione originaria (original position). Tuttavia, a favore dei due principi (e, in particolare, del secondo) viene formulato anche un argomento intuitivo, indipendente dalla dimostrazione via teoria della scelta. L'argomento intuitivo esemplifica i principali impegni della visione pre-analitica, come avrebbe detto Schumpeter, destinati a modellare la costruzione concettuale della teoria.

Su questo sfondo, mi propongo in primo luogo di mettere a fuoco i) la connessione fra il principio di libertà e il principio di differenza e ii) l'interpretazione del secondo principio in termini di eguaglianza democratica, offerta dall'argomento intuitivo.

In secondo luogo, mi soffermo iii) sull'interpretazione della posizione originaria come situazione di scelta appropriata per gli scopi della concezione della giustizia come equità e iv) sviluppo qualche cenno su alcuni casi di possibile estensione della teoria all'equità intergenerazio-

1 Cfr. J. Rawls, A Theory of Justice, Cambridge, Mass., Harvard University Press, 1971. 
nale, alle questioni lato sensu ambientali e all'arena internazionale, in cui si formulano i dilemmi di una teoria della giustizia globale.

\section{L'EQUITÀ SOCIALE E IL VALORE DELLA LIBERTÀ}

Chiediamoci ora: qual è il rapporto fra il primo principio di libertà eguale e il secondo principio destinato a modellare la distribuzione di beni sociali primari come beni di cittadinanza? La domanda è importante perché i due principi, nel loro ordinamento, specificano l'interpretazione dell'equità sociale.

Risponderei così alla domanda: l'avere le persone eguale libertà non implica che sia eguale per le persone il valore della loro eguale libertà. Il maggiore o minore valore dell'eguale sistema delle libertà fondamentali dipenderà da qualcosa che non ha a che vedere con la libertà, quanto con la distribuzione di costi e benefici della cooperazione sociale che miri a equalizzare, per quanto è possibile, il valore della libertà per le persone. L'equità nell'accesso ai beni sociali primari è richiesta dalla necessità di mantenere la promessa dell'eguale libertà per chiunque. Vantaggi e svantaggi nell'accesso ai beni sociali primari sono infatti responsabili della variazione in positivo o in negativo del valore della libertà.

Qui siamo al cuore della questione sociale, vecchia e nuova, che chiede principi di giustizia, alla luce di un'idea di eguale cittadinanza democratica. Il giovane Marx aveva fissato i termini della questione nel suo scritto Sulla questione ebraica, distinguendo fra il cielo degli eguali diritti di citoyenneté e la terra delle ineguaglianze del bourgeois e denunciando in tal modo la comunità degli eguali diritti come "comunità illusoria”.

Si osservi che un modo per rendere conto del significato dell'equità in una teoria della giustizia sociale è quello che la connette all'eguale status di cittadinanza democratica, all'eguale considerazione e rispetto dovuto a ciascun cittadino e a ciascuna cittadina, per usare la terminologia del compianto Ronald Dworkin².

Questa considerazione mi consente di introdurre il secondo punto, che coincide con l'analisi dell'argomento intuitivo a favore del

2 Cfr. R. Dworkin, Sovereign Virtue: the Theory and Practice of Equality, Cambridge, Mass., Harvard University Press, 2000. 
principio di differenza, di cui ho cercato di mostrare la connessione stretta con il principio di eguale libertà.

\section{L'ARGOMENTO INTUITIVO E L'EGUAGLIANZA DEMOCRATICA}

Per illustrare l'argomento intuitivo, consideriamo come differenti modelli di credenza e valore politico, familiari nella nostra tradizione, offrano una distinta interpretazione dell'equità nella distribuzione. Formuliamo un principio generale di equità distributiva $\mathrm{o}$, come si dice, una norma sociale di equità, distinta da una norma di eguaglianza stretta. Diciamo che tutti i valori sociali devono essere distribuiti egualmente a meno che una ineguaglianza nella loro distribuzione non vada i) a vantaggio di ciascuno, ii) in presenza di carriere aperte ai talenti. Come vedremo, le differenti interpretazioni vertono sulle due clausole.

Sono a disposizione almeno tre modelli di interpretazione politica. Li chiamiamo, sulla scorta della terminologia di Rawls, il sistema della libertà naturale, l'eguaglianza liberale e l'eguaglianza democratica. Ora, il sistema della libertà naturale e l'eguaglianza liberale interpretano la prima clausola "a vantaggio di ciascuno" applicando il principio di efficienza, un principio paretiano adattato al caso. Data una distribuzione iniziale di vantaggi e svantaggi, sarà nell'interesse collettivo (e quindi giusta) qualsiasi soluzione efficiente. (Qualsiasi altra distribuzione, infatti, danneggerebbe almeno uno, avvantaggiato o svantaggiato.)

Diversa è invece l'interpretazione che viene favorita della seconda clausola sulle carriere aperte ai talenti. Il modello della libertà naturale la specifica come eguaglianza formale. Il modello dell'eguaglianza liberale, come eguaglianza di opportunità. E ciò, perché si avverte che la distribuzione iniziale di dotazioni sociali è, da un punto di vista etico, intrinsecamente arbitraria: è come l'esito di una lotteria sociale (nessuno, del resto, sceglie di nascere né, a fortiori, da chi e dove e quando nascere).

Per contrasto, il modello dell'eguaglianza democratica, mentre adotta questa interpretazione della seconda clausola, specifica la prima sostituendo al principio di efficienza paretiana il principio di differenza. Esso afferma che il vantaggio di ciascuno va inteso, prioritariamente, come il vantaggio di chi è sfavorito nella distribuzione e nell'accesso ai beni sociali primari. Il principio di differenza è sensibile agli effetti moralmente arbitrari sia della lotteria sociale sia della lotteria naturale. E lavora congiuntamente con l'equa eguaglianza delle opportunità. Il 
modello dell'eguaglianza democratica ci chiede di valutare le distribuzioni di valori sociali adottando la prospettiva dell'eguale cittadinanza e dà priorità a chi è, in esse, più svantaggiato.

Si osservi che, come hanno mostrato Derek Parfit, in un celebre articolo, e Thomas Nagel nel capitolo sull'eguaglianza in Mortal Questions, l'egualitarismo democratico è in effetti prioritarismo (priorità all'urgenza dello svantaggio) e, come ha mostrato Thomas Scanlon in un altro saggio ormai classico, l'idea di equità implica che i termini equi della cooperazione nel tempo debbano essere accettabili, o ragionevolmente non rifiutabili, in primo luogo, da parte di coloro per cui essi sono meno accettabili³.

Equo sarà, quindi, uno schema di cooperazione nel tempo i cui termini fondamentali devono poter essere accettati da chiunque; prioritariamente da chi è, senza sua responsabilità, sfavorito dalla sorte naturale e sociale. L'intuizione di base è quella secondo cui il fatto radicale dell'ingiustizia sociale comincia con le storie di bambine e bambini, il cui piano di vita sia modellato dall'arbitrarietà morale di una nascita, qua e là per il mondo.

La concezione della giustizia come equità presuppone una schema di reciprocità nel tempo, che sia inclusivo nei confronti di chiunque - in quanto cittadino e cittadina - sia degno di eguale considerazione e rispetto. Questo esito, che è ascrivibile all'argomento intuitivo, deve ora essere dimostrato nel quadro analitico della teoria della scelta in posizione originaria. Vedremo come in questo quadro ritroveremo alcuni dei punti essenziali, già messi in luce a proposito dell'idea di equità specificata dalla teoria contrattualistica della giustizia sociale.

\section{L'ESPERIMENTO MENTALE DELLA POSIZIONE ORIGINARIA}

Come ho accennato, la teoria della giustizia come equità si basa su un celebre e controverso argomento che è chiamato: posizione origina-

3 Cfr. D. Parfit, Equality and Partiality, Ratio, 10, 3 (1997), 202-221; T. Nagel, Mortal Questions, Cambridge, Cambridge University Press, 1979, cap. 8; T. Scanlon, Preference and Urgency, The Journal of Philosophy, XIX (1975), 655-669. Mi sia consentito rinviare, in proposito, al mio L'immaginazione filosofica e altri saggi, Milano, Feltrinelli, 2012, 104-125. 
ria. L'idea è più o meno la seguente: immaginiamo di dover giustificare mutuamente quali siano i termini equi per la cooperazione fra noi nel tempo. Quale distribuzione di diritti e doveri, di costi e benefici sia fra quelle possibili - quella che riconosceremmo come equa. Quale intesa cooperativa superi il test dell'equità fra le varie intese cooperative razionalmente possibili. Quale contratto sociale sia selezionabile come equo sulla curva dei contratti, come esiti di giochi di contrattazione.

$\mathrm{Si}$ propone allora di procedere con un esperimento mentale, con una ipotesi che mira semplicemente a chiarire la natura del problema di scelta collettiva (nella giustizia come equità, il problema della giustificazione si riformula nei termini di un problema di deliberazione. E in questo senso preciso la teoria è contrattualistica). Supponiamo di trovarci in una situazione di scelta fra principi alternativi di giustizia, in cui la nostra informazione su noi stessi sia ridotta e significativamente incompleta. Non sappiamo chi siamo, quali dotazioni e interessi siano i nostri, quali scopi e concezioni del bene noi abbiamo, quale posto la sorte naturale e sociale ci abbia assegnato nello schema di cooperazione sociale. Siamo, come si usa dire, "sotto un velo di ignoranza". Sappiamo di avere scopi, ma non sappiamo quali siano. Sappiamo di avere bisogni, ma non sappiamo quali siano. Sappiamo di essere nati da qualche parte, ma non sappiamo dove. Sappiamo di essere cresciuti in un certo ambiente, ma non sappiamo in quale ambiente. E così via. Dobbiamo scegliere impersonalmente i termini equi della nostra convivenza quali cittadini e cittadine: i principi di giustizia come equità, selezionati entro una lista di alternative.

Ora, la teoria sostiene che individui sotto un velo di ignoranza convergerebbero sulla scelta di uno schema di cooperazione equo e accettabile (o non rifiutabile) per chiunque, e che l'equità dei termini della cooperazione, specificata dai due principi di giustizia, dipenderebbe esclusivamente dall'equità della situazione iniziale di scelta, interpretata come posizione originaria. Ciascuno di noi, avrebbe detto Rousseau, è chiunque. O meglio, sceglie dal punto di vista di ciascun altro. Equo sarà lo schema di cooperazione scelto, adottando il punto di vista di chiunque. E, data la condizione di simmetria, ottenuta grazie ai vincoli sull'informazione della posizione originaria, si può dimostrare che ciascuno converge su una soluzione di equità, adottando il punto di vista dell'individuo rappresentativo del gruppo più svantaggiato.

Si osservi: in condizioni di rischio, non sapendo io se sono avvantaggiato o svantaggiato, è razionale mi assicuri quanto alla probabilità, la cui stima soggettiva è preclusa, che la mia sorte sociale sia svantaggia- 
ta (la logica della scelta richiama un'analogia con la regola di maximin nei casi di rischio). Nella riformulazione del maximin come leximin, proposta nei suoi classici lavori di teoria della scelta sociale da Amartya Sen, diremo che uno schema di cooperazione sociale, per essere equo per i partecipanti, deve poter essere accettato prioritariamente dall'individuo rappresentativo del gruppo più svantaggiato, secondariamente dall'individuo rappresentativo del gruppo immediatamente meno svantaggiato, e così via. Quindi, possiamo concludere, riconoscere l'equità di istituzioni, provvedimenti e pratiche sociali vuol dire accettare una sorta di potere di veto morale da chi, senza sua responsabilità, è più sfavorito dalla sorte naturale e sociale.

Non è difficile riconoscere in che senso questo esito vede convergere sia l'argomento intuitivo sia la costruzione analitica del contratto sociale in posizione originaria. Visione pre-analitica e costruzione teorica della giustizia come equità pervengono, secondo Rawls, a un esito di equilibrio riflessivo.

\section{CASI DI ESTENSIONE E L'IDEA DI GIUSTIZIA GLOBALE}

Vorrei conclusivamente richiamare almeno tre casi di estensione della concezione della giustizia come equità. Il primo è quello della giustizia fra generazioni, che Rawls discute a lungo nel $\$ 44$ di $A$ Theory of Justice, dedicato al principio del giusto tasso di risparmio. Il secondo caso di estensione è solo accennato nell'ultima parte del $\$ 77$ sulle basi dell'eguaglianza e concerne i nostri doveri nei confronti degli animali non umani e della natura. Il terzo caso è quello dell'estensione della giustizia come equità all'arena internazionale, discusso nel $₫ 58$, dedicato alla giustificazione dell'obiezione di coscienza.

La concezione di giustizia come equità presuppone il riferimento a una singola comunità politica, delimitata da confini. Rawls non ha mai rinunciato a questa assunzione che definisce la portata e i limiti di applicazione della sua idea di equità. Il più impegnativo contributo teorico all'estensione della teoria all'arena internazionale coincide con il suo ultimo libro, The Law of Peoples, uscito nel $1999^{4}$.

4 Cfr. J. Rawls, The Law of Peoples, Cambridge, Mass., Harvard University Press, 1999. 
Rawls propone, in una prospettiva ispirata al federalismo kantiano di Per la pace perpetua, una duplice versione della posizione originaria che includa parti rappresentative di società liberali e di società non liberali ma bene ordinate, per selezionare i principi di un diritto internazionale fin de siècle. Negli ultimi vent'anni il problema cruciale per la filosofia politica è stato ed è tuttora costituito dal rompicapo dell'estensione. Giustizia come equità resta una sorta di terminus a quo per la ricerca. Sembra si possa dire che dovremmo poter riformulare l'idea di equità non più o non solo nei termini di giustizia internazionale, ma anche e propriamente nei termini di una prospettiva plausibile di giustizia come equità globale, che incorpori le dimensioni plurali della sostenibilità.

Tutta la faccenda dell'estensione è terribilmente difficile, quando si abbandoni il riferimento a una singola comunità politica e all'idea connessa di eguale cittadinanza. Ma l'idea di giustizia globale, come ha efficacemente sostenuto Amartya Sen, resta per noi ineludibiles.

5 Cfr. A. Sen, The Idea of Justice, London, Penguins Books, 2009. Ho affrontato alcuni dilemmi di una teoria della giustizia globale a partire da La bellezza e gli oppressi, Milano, Feltrinelli, 2002 sino a L'immaginazione filosofica e altri saggi, 2012, cit. 